\section{Fascitis necrosante secundaria a onfalitis en un recién nacido con hipoplasia del bazo}

\section{Necrotizing fascitis due to omphalitis in a newborn with hypoplastic spleen}

\author{
Karla Santos-Jasso ${ }^{1}$ \\ Cecilia Ridaura-Sanz ${ }^{2}$ \\ ${ }^{1}$ Adscrita al Servicio de Cirugía General. \\ 2 Jefe Servicio de Patología Post mortem. Departa- \\ mento de Patología. \\ Instituto Nacional de Pediatría.
}

\section{PRESENTACIÓN}

Se presenta el caso de una recién nacida con hipoplasia del bazo que desarrolló fascitis necrosante de la pared abdominal secundaria a onfalitis.

\section{RESUMEN CLÍNICO}

Niña de 18 días de vida producto de segundo embarazo, obtenida de término (38 semanas de gestación) por parto eutócico, con peso al nacer de 3105 g y Apgar 9/9. Fue egresada como binomio sano a las 12 horas de vida extra uterina.

La primera evacuación meconial se presentó en las primeras 24 horas de vida; sin embargo, los padres refirieron dificultad para evacuar desde el nacimiento. Cuarenta y ocho horas previas a su ingreso le realizaron masaje abdominal y administraron aceite de oliva e infusión de manzanilla por vía oral. En las siguientes horas la paciente mostró hiporexia y ausencia de evacuaciones fecales por lo que un médico inició tratamiento con laxante no especificado y dimeticona. Posteriormente, al no presentar mejoría y agregarse irritabilidad y distención abdominal, acudió a nuestra institución.

A la exploración física inicial se registró peso de 3850 g, talla de $48 \mathrm{~cm}$, frecuencia cardíaca de 180 latidos por minuto, frecuencia respiratoria de 58 respiraciones por minuto, presión arterial de $92 / 72 \mathrm{mmHg}$ y temperatura de $39.5^{\circ} \mathrm{C}$. Se encontró irritable, bien hidratada, con palidez generalizada, cráneo normocéfalo, fontanela anterior normotensa, rasgos faciales simétricos, cardio-pulmonar sin alteraciones, abdomen globoso
Recibido: 9 de diciembre del 2014

Aceptado: 10 de diciembre del 2014

Correspondencia: Dra. Cecilia Ridaura Sanz Jefe Servicio de Patología Post mortem Departamento de Patología Instituto Nacional de Pediatría Insurgentes Sur 3700-C CP 04530 México, D.F. cridaura@gmail.com

Este artículo debe citarse como Santos Jasso K, Ridaura Sanz C. Fascitis necrosante secundaria a onfalitis en un recién nacido con hipoplasia del bazo. Acta Pediatr Mex 2015;36:36-42. 
con perímetro abdominal de $41 \mathrm{~cm}$, con salida de líquido transparente escaso por la región umbilical, ruidos peristálticos ausentes, dolor intenso a la palpación superficial, extremidades simétricas y móviles, Ilenado capilar en dos segundos. El ano permeable y dentro del complejo muscular anorrectal; al tacto rectal tono conservado con evacuaciones explosivas y abundante salida de gas rectal. Se colocó sonda rectal y se realizó irrigación colónica con mejoría parcial de las condiciones abdominales. La radiografía de abdomen mostró edema de pared abdominal con distribución de asas intestinales centrales, no se observó distensión intestinal ni neumatosis intestinal (Figura 1). Se dejaron sondas orogástrica y rectal. Se canalizó vena periférica y se inicio tratamiento con cefotaxima, metronidazol, paracetamol, buprenorfina y se ingresó al servicio de neonatología. La biometría hemática reportó una hemoglobina de $15.1 \mathrm{~g} /$ $\mathrm{dL}$, hematocrito de $44.9 \%$, leucocitos 20800



Figura 1. Radiografía abdominal: distribución de asas intestinales centrales y edema de pared abdominal. células $/ \mathrm{mm}^{3}$ con neutrófilos de $58 \%$ y plaquetas de $229000 / \mathrm{mm}^{3}$; sodio de $128 \mathrm{mEq} / \mathrm{L}$, potasio de $5.8 \mathrm{mEq} / \mathrm{L}$, cloro de $105 \mathrm{mEq} / \mathrm{L}$, calcio $7.8 \mathrm{mg} /$ $\mathrm{dL}$, gasometría venosa con $\mathrm{pH}$ de $7.39, \mathrm{PCO}_{2}$ de $26 \mathrm{mmHg}, \mathrm{HCO}_{3} 15.5 \mathrm{mg} / \mathrm{dL}$, déficit de base de -9.4, lactato de $5.76 \mathrm{mmol} / \mathrm{L}$. Se administró una carga de líquidos intravenosa a $20 \mathrm{~mL} / \mathrm{kg}$ e inicio de infusión de soluciones parenterales; se repitió control gasométrico después de la hidratación con $\mathrm{pH} 7.36, \mathrm{PCO}_{2}$ de $33 \mathrm{mmHg}, \mathrm{HCO}_{3}$ de $18.7 \mathrm{mg} /$ $\mathrm{dL}$, déficit de base -5.5 y lactato de $2.26 \mathrm{mmol} / \mathrm{L}$.

A 20 horas de su ingreso hospitalario se incrementó la distensión abdominal, hubo dolor intenso a la palpación en la región periumbilical y se observó edema con calor local y aspecto de piel de naranja (Figura 2); el control gasométrico mostró $\mathrm{pH}$ de 7.22 , con $\mathrm{PCO}_{2}$ de $35 \mathrm{mmHg}$,

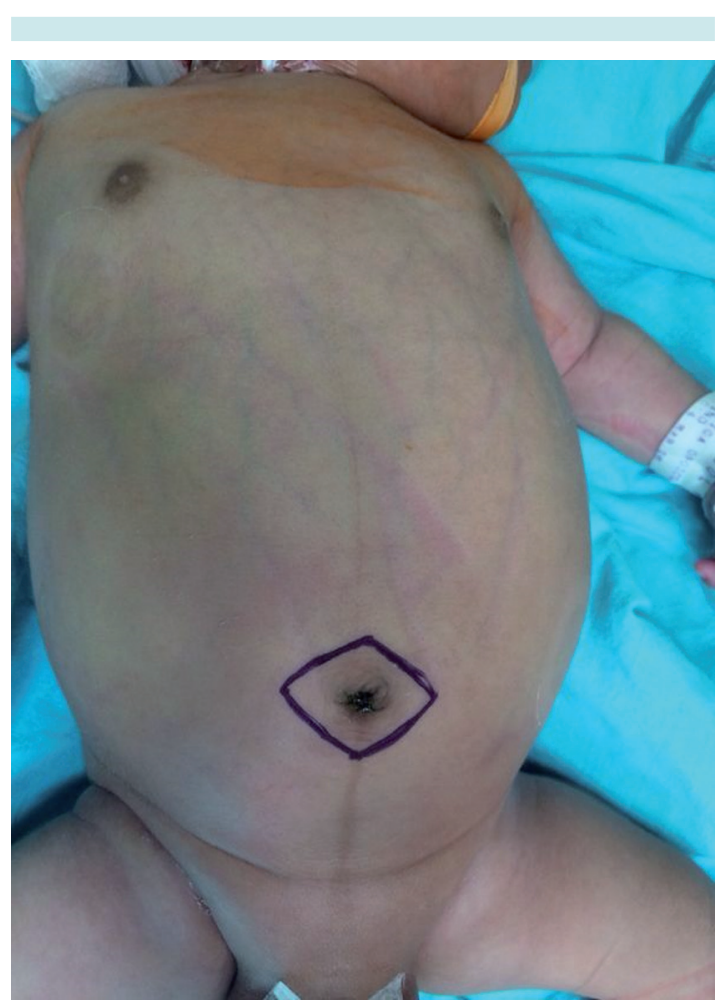

Figura 2. Distensión abdominal, eritema periumbilical, edema local y aspecto de piel de naranja. 
$\mathrm{HCO}_{3}$ de $13.8 \mathrm{mg} / \mathrm{dL}$, déficit de base -14 con lactato de $3.0 \mathrm{mmol} / \mathrm{L}$. Se decidió realizar exploración quirúrgica abdominal por sospecha de fascitis necrosante de la pared abdominal probablemente asociada con onfalitis; se incrementó la cobertura antimicrobiana contra enterobacterias productoras de betalactamasas, Pseudomonas sp y gérmenes anaerobios con meropenem y teicoplanina. En la laparotomía exploradora se encontraron $100 \mathrm{~mL}$ de líquido libre en la cavidad peritoneal, con intestino normal y sin hallazgos patológicos dentro de la cavidad peritoneal; se realizó resección de la cicatriz y de los vasos umbilicales con tejido circundante aproximadamente $3 \mathrm{~cm}$ a cada lado de la cicatriz umbilical (Figura 3 ) y se colocó un sistema cerrado de presión negativa (vacuum assisted closure, VAC) para el cierre de la pared abdominal y se colocó un catéter venoso central.

Egresó del quirófano intubada con parámetros de ventilación en modo CMV con PI de $19 \mathrm{~cm} /$ $\mathrm{H}_{2} \mathrm{O}$, PEEP de $4 \mathrm{~cm} / \mathrm{H}_{2} \mathrm{O}, \mathrm{FIO}_{2}$ de $75 \%$. La acidosis metabólica continúo incrementándose con hiperlactatemia; tuvo hipotensión arterial con hipoperfusión tisular y anuria; se inició manejo con aminas vasopresoras: dopamina $10 \mu \mathrm{g} / \mathrm{kg} /$

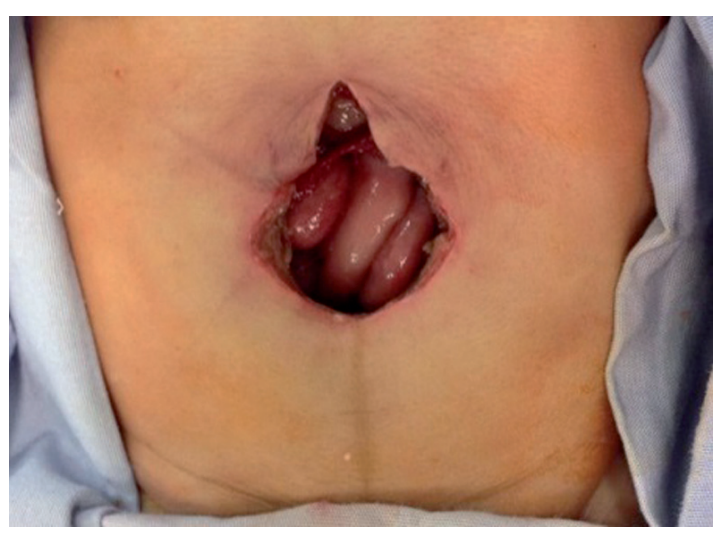

Figura 3. Resección de la cicatriz y vasos umbilicales; hallazgo de contenido intestinal de aspecto normal. min y dobutamina $10 \mu \mathrm{g} / \mathrm{kg} / \mathrm{min}$. Desarrolló hiperpotasemia grave $(7 \mathrm{mEq} / \mathrm{L})$ e hiperglicemia con gran fuga capilar y edema generalizado; se agregó adrenalina $(0.1 \mu / \mathrm{kg} / \mathrm{min})$. Tuvo bradicardia de 60 latidos por minuto, se iniciaron maniobras de reanimación cardiopulmonar sin éxito y falleció.

\section{COMENTARIO CLÍNICO}

Recién nacida eutrófica y con el antecedente de dificultad para presentar evacuaciones fecales; según los padres un patrón de evacuaciones de 1 cada 72 horas aproximadamente y sólo con estimulación rectal. El padecimiento agudo con datos pivote de distensión abdominal e irritabilidad, integraron inicialmente un síndrome doloroso abdominal con aparente patrón obstructivo intestinal, iniciándose abordaje terapéutico y diagnóstico de enfermedad de Hirschsprung, por lo que se realizaron descompresión rectal y colónica con una sonda transrectal e irrigaciones. La ausencia de evacuaciones fecales y la salida de gas y evacuaciones fecales explosivas después de la colocación de una sonda transrectal fueron un gran distractor; sin embargo, el control radiológico abdominal no mostró un patrón obstructivo intestinal y sí un manifiesto edema de pared abdominal. El hallazgo de líquido seroso umbilical escaso a su ingreso y el posterior eritema periumbilical fundamentaron el diagnóstico de onfalitis con datos clínicos infecciosos (fiebre, respuesta inflamatoria sistémica con foco identificado en la pared abdominal) que se complicó con una franca acidosis metabólica e hipoperfusión tisular. Los datos abdominales periumbilicales asociados con los datos de sepsis grave llevaron a la sospecha diagnóstica de fascitis necrosante de la pared abdominal que requería de desbridamiento quirúrgico.

Podemos concluir que tras el análisis clínico del caso se integraron los diagnósticos sindrómicos de: 
- Sindrome infeccioso (fiebre y datos inflamatorios locales en la región periumbilical).

- Síndrome de sepsis grave (respuesta inflamatoria sistémica con foco infeccioso en la pared abdominal y evidencia de disfunción orgánica).

\section{COMENTARIO ANATOMOPATOLÓGICO}

El estudio histopatológico del ombligo resecado presentó la piel ulcerada con un exudado inflamatorio de polimorfonucleares con flebitis de la vena umbilical (Figura 4). El exudado se extendió a la fascia profunda con necrosis de los tejidos blandos de la pared abdominal (Figura 5). La tinción de Brown-Brenn reveló la presencia de bacterias grampositivas (Figura 6). Las arterias umbilicales tenían calcificación de la pared y el uraco fue histológicamente normal. Con estos datos se estableció el diagnóstico de onfalitis aguda y fascitis necrosante de la pared abdominal.

El estudio post mortem mostró datos de septicemia con hepatitis séptica y datos de choque con

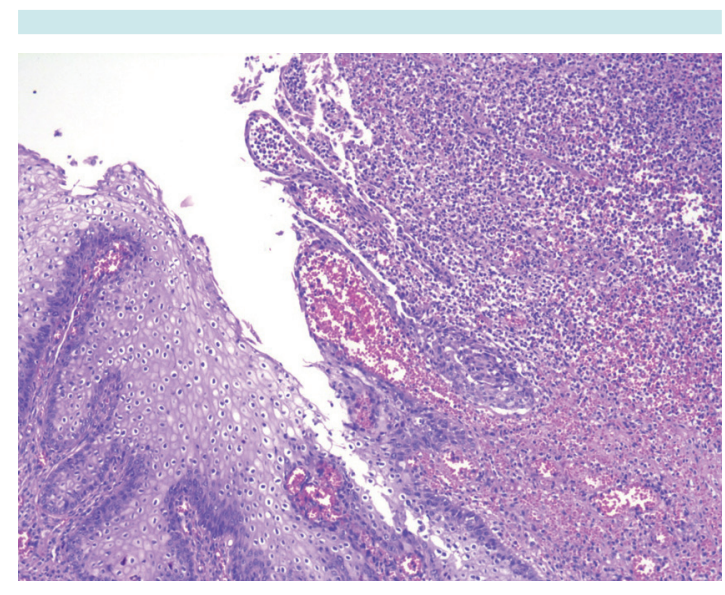

Figura 4. Corte histológico del ombligo: epitelio ulcerado y reemplazado por denso infiltrado inflamatorio y proliferación vascular.

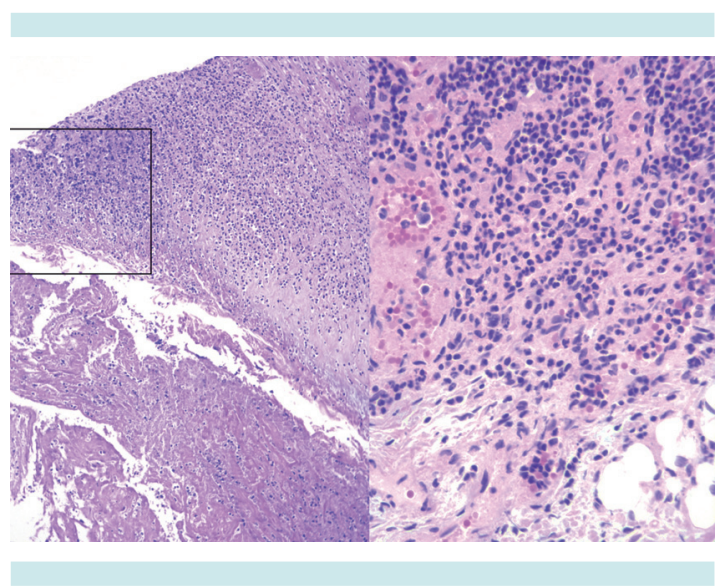

Figura 5. Corte histológico de la fascia profunda y pared abdominal: necrosis del tejido y exudado inflamatorio (recuadro).

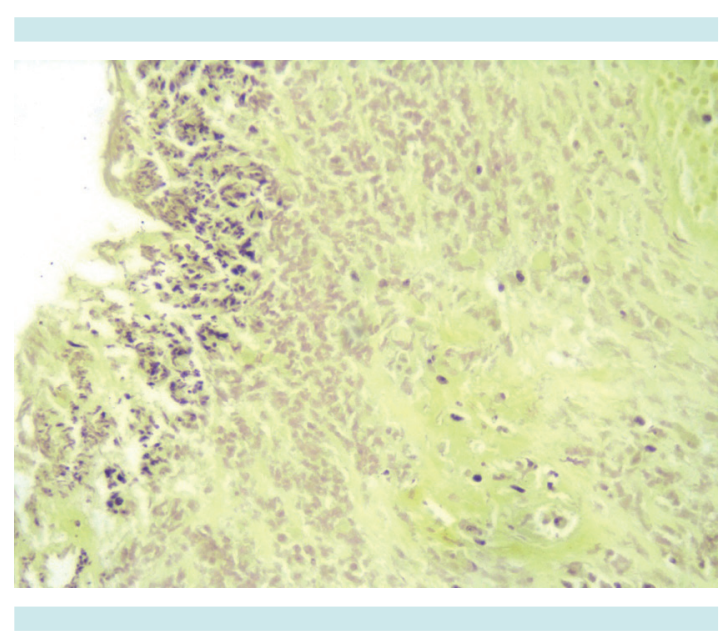

Figura 6. Tinción de Brown-Brenn: bacterias grampositivas.

hemorragia multifocal y necrosis tubular aguda incipiente. El pulmón con múltiples hemorragias parenquimatosas recientes aún presentaba escamas amnióticas en la luz alveolar en forma focal. El intestino levemente dilatado en la porción ascendente del colon con plexos mientéricos normales en toda la extensión.

El timo se encontró disminuido levemente de tamaño e histológicamente con datos de 
estrés agudo, necrosis de linfocitos corticales y numerosos macrófagos con fagocitosis de restos celulares. Hubo corpúsculos de Hasall en calidad y número que permitieron descartar la posibilidad de displasia tímica. El resto del tejido linfoide no tuvo alteraciones importantes. La médula ósea tenía celularidad normal.

El único dato que pudo considerarse como un factor de riesgo para desarrollar esta grave complicación infecciosa fue la gran disminución del tamaño y peso del bazo (5.56 g vs. 10 g) (Figura 7). Si además, histológicamente, presentaba datos de esplenitis séptica y congestión de la pulpa roja, situaciones que ocasionan esplenomegalia, suponemos que originalmente el peso debió haber sido menor.

La disminución del tamaño del bazo y su ausencia han sido asociados con la insuficiencia de este órgano conocida como hipoesplenismo y que se define como una disminución o abolición de las dos funciones básicas del bazo: 1) Filtrado de eritrocitos disfuncionales; 2) Combate de la infección.

El fenotipo clínico suele ser el de una abrumadora septicemia, generalmente por organismos

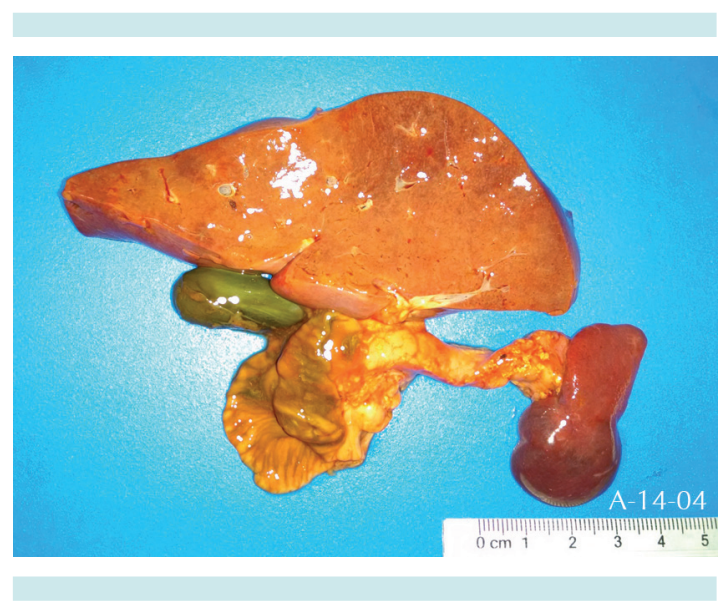

Figura 7. Hipoesplenia. encapsulados. En este caso la etiología de la infección no fue determinada por cultivo ya que en vida no se realizaron y en el estudio post mortem los órganos cultivados fueron negativos (líquido cefalorraquídeo, bazo, hígado y pulmón). Sin embargo, las colonias bacterianas grampositivas en la lesión umbilical apoyan la suposición de que este tipo de gérmenes haya sido el causante de la fascitis necrosante. El diagnóstico de hipoesplenismo requiere la demostración de la insuficiencia funcional del bazo, lo cual desafortunadamente no se documentó. Es importante tener en cuenta este factor de riesgo potencial en la infección neonatal. Existen algoritmos para la detección de este problema.

El ultrasonido abdominal permite el diagnóstico de asplenia o de hipoesplenia y, una vez detectada la alteración anatómica, proceder a la evaluación funcional. Hay diferentes pruebas para esta evaluación. En un frotis de sangre periférica pueden encontrarse eritrocitos anormales con irregularidades en la membrana o cuerpos de Howell-Jolly, que son remanentes nucleares de los eritrocitos. Otro estudio más específico es el escaneo con partículas marcadas con tecnecio 99m para evaluar la captación esplénica de las mismas. ${ }^{1-5}$

\section{DIAGNÓSTICOS ANATÓMICOS}

Onfalitis aguda

Fascitis necrosante periumbilical

Estado después de la resección de ombligo y uraco (8 horas)

Septicemia

Hepatitis séptica

Involución aguda del timo

Datos anatómicos de choque

Miopatía isquémica en músculo liso intestinal

Necrosis tubular aguda incipiente

Congestión y hemorragias recientes multiviscerales

Hipoplasia esplénica (probable hipoesplenismo) 


\section{DISCUSIÓN}

La fascitis necrosante abdominal en el período neonatal generalmente es secundaria a una infección cutánea, principalmente onfalitis; otras infecciones cutáneas descritas han sido balanitis, mastitis, complicaciones posoperatorias o secundarias al uso de un catéter umbilical. Otras causas referidas han sido inmunodeficiencias, enterocolitis necrosante, impétigo ampolloso y lesión de electrodo colocado en región del cuero cabelludo, seguidas de una circuncisión con un dispositivo de plastibell, mastitis materna e infección de tractos urinarios persistentes permeables o remanentes del conducto onfalomesentérico.

En el caso en discusión probablemente el curso de una onfalitis no diagnosticada ni tratada, asociada a una probable inmunodeficiencia celular derivada de un hipoesplenismo, podría ser la causa del problema. Las causas de onfalitis más frecuentes como parto prolongado, infección amniótica, prematuridad, bajo peso al nacer, aplicación de sustancias en el ombligo, defectos en las moléculas de adhesión y motilidad leucocitaria y defectos en la producción del interferón no parecían estar presentes como factores de riesgo. Por otra parte, el diagnóstico de persistencia de uraco se descartó en el transquirúrgico y en el estudio histopatológico.

Las posibles complicaciones de una onfalitis sin tratamiento adecuado pueden ser septicemia, abscesos intrabdominales, peritonitis, obstrucción intestinal adhesiva, tromboflebitis de las venas hepáticas, evisceración espontánea del intestino delgado y fascitis necrosante.

La fascitis necrosante es una infección bacteriana grave de los tejidos blandos, con mayor prevalencia en los adultos. En niños es una en- fermedad poco frecuente pero con un desenlace fulminante y alta mortalidad, especialmente en la etapa neonatal. Se caracteriza por un marcado edema, rápida progresión de la inflamación e involucramiento séptico sistémico de alta letalidad. ${ }^{6}$

Hay descripciones de casos de fascitis necrosante desde 1924, pero el término fue introducido en 1952 por Wilson, quien describió una inflamación progresiva con necrosis del tejido celular subcutáneo, fascia superficial y profunda con gangrena cutánea. ${ }^{7}$ El sitio más común es la pared abdominal seguida por el tórax anterior o posterior, las extremidades, regiones escrotal y perianal. $.6,8,9$

La presentación inicial muestra en la piel eritema, edema o celulitis. La lesión evoluciona rápidamente y puede avanzar a una coloración violácea o tener el aspecto de piel de naranja (edema importante de todo el tejido celular subcutáneo), mostrar bulas o vesículas y progresar a necrosis. La crepitación es poco común. Los datos de respuesta inflamatoria sistémica siempre están presentes (fiebre, taquicardia), la cuenta leucocitaria sanguínea siempre es elevada con bandemia, la trombocitopenia está presente en la mitad de los casos reportados. Los hemocultivos tienen aislamiento polibacteriano en aproximadamente la mitad de los casos reportados ${ }^{6}$ y se han publicado aislamientos de Staphylococcus aureus adquiridos en la comunidad y resistentes a la meticilina. ${ }^{10-13}$

El tratamiento requiere de antimicrobianos y desbridamiento quirúrgico extenso temprano de la fascia involucrada. Los principios quirúrgicos siguen vigentes para esta patología desde 1924 y son: escisión del tejido necrosado hasta donde exista extensión del mismo dejando tejido viable y reexploraciones quirúrgicas frecuentes de ser necesarias. ${ }^{14}$ Hay reportes de caso con uso de oxígeno hiperbárico; sin embargo, esos pacientes 
tuvieron desenlaces fatales y se desconoce si es por la misma evolución patológica de la enfermedad o por el uso de la terapia hiperbárica. El uso intravenoso de inmunoglobulinas polivalentes es controvertido. ${ }^{12}$ La tasa de mortalidad reportada ha sido hasta de $59 \%$. Los sobrevivientes reportados han mostrado grandes defectos abdominales con pobre granulación ${ }^{6}$ y requieren terapias prolongadas. La reconstrucción suele requerir de piel artificial. ${ }^{15}$

\section{CONCLUSIÓN}

En este caso los datos clínicos de distensión abdominal sin patrón obstructivo intestinal, asociados con onfalitis que evolucionó a una sepsis grave con involucramiento multiorgánico permitieron sospechar el diagnóstico de fascitis necrosante abdominal, una infección bacteriana de tejidos blandos rara y con desenlace fatal en la mayoría de los casos reportados. En la etapa neonatal algunos casos de fascitis necrosante han sido atribuibles a infecciones cutáneas como onfalitis, balanitis o complicaciones posoperatorias. El alto índice de sospecha, el desbridamiento quirúrgico temprano, el uso de antimicrobianos sistémicos de amplio espectro, soporte hemodinámico y cuidados intensivos neonatales, en conjunto, son indispensables para lograr la supervivencia de estos pacientes. En este caso, ante la ausencia de otros factores de riesgo, suponemos que la hipoplasia del bazo fue la afección insospechada que contribuyó a la complicación fatal de la onfalitis.

\section{REFERENCIAS}

1. Frasse N, Davies BW, Cusack J. Neonatal omphalitis. A review of its serious complications. Acta Paediatr 2006;95:519-22.

2. Ulloa-Gutiérrez R, Rodríguez-Calzada H, Quesada L, Arguello A, Ávila Aguero M. Is it a cute omphalitis or necrotizing fasciitis. Report of three fatal cases. Pediatr Emerg Care 2005;21:600-2.

3. William BM, et al. Hypoesplenism: A comprehensive review Part 1: Basic concepts and causes. Hematology 2007;12;1-13.

4. William BM, et al. Hypoesplenism A comprehensive review Part II Clinical manifestations, diagnosis and management. Hematology 2007;12:89-98.

5. Lammers ALJ, et al. Hypoesplenismo: Comparison of different methods for determining splenic function. Am J Hematol 2012;87:484-489.

6. Hsieh WS, Yang PH, Chao HC, Lai JY. Neonatal necrotizing fasciitis: A report of three cases and review of the literatura. Pediatrics 1999;103(4):e53.

7. Wilson B. Necrotizing fasciitis. Am Surg 1952;18(4):416-31.

8. Zgraj O, Paran S, O'Sullivan M, Quinn F. Neonatal scrotal Wall necrotizing fasciitis (Fournier gangrene): a case report. J Med Case Rep 2011;5:576.

9. Christensen RD, Pysher TJ, Christensen SS. Case report: perianal necrotizing fasciitis: in a near-term neonate. J Perinatol 2007;27(6):390-1.

10. Hayani KT, Mathew R, Oyedele T, Hulten KG. Neonatal necrotizing fasciitis due to community acquired methicilin reistant Staphylococcus aureus. Pediatr Infect Dis J 2008;27(5):480-1.

11. Gangopadhyay AN, Pandey A, Upadhyay VD, Sharma SP, Gupta DK, Kumar V. Neonatal necrotising fasciitis - Varanasi experience. Int Wound J 2008;5(1):108-12.

12. Faye $\mathrm{A}$, Lorrot $\mathrm{M}$, Bipet $\mathrm{P}$, Bonacorsi $\mathrm{S}$, Cohen R. Managment of severe invasive group A streptococcal infections. Arch Pediatr. 2014;21(S2):S87-S92. Doi: 10.1016/S0929-693X(14)72267-5.

13. Prachukthum S. Kositamongkol S, Bunjoungmanee $P$. Tangsathapornpong A. Necrotizing fasciitis: arare manifestation of late-onset neonatal group B streotococcal infection. J Med Assoc Thai 2014;9(6):669-72.

14. Meleney FL. Hemolytic streptococcus gangrene. Arch Surg 1924;9:317-364.

15. Mazzone L, Schiestl C. Managment of septic skin necroses. Eur J Pediatr Surg 2013;23(5):349-58. 